\title{
Subarachnoid haemorrhage in Plasmodium falciparum malaria
}

\author{
K. Murugavel, S. Saravanapavananthan, A. Anpalahan and R.F. James
}

Medical Units 16B \& 18B, General Hospital - Teaching, Jaffna, Sri Lanka.

\begin{abstract}
Summary: A 41 year old man with cerebral malaria was found to have numerous bilateral retinal haemorrhages and very high parasitaemia. Despite intensive treatment his condition deteriorated and he died. Autopsy showed subarachnoid haemorrhage, which has not been previously described in cerebral malaria.
\end{abstract}

\section{Introduction}

Cerebral malaria is one of the pernicious syndromes seen in Plasmodium falciparum malaria. It can lead to hemiplegia, convulsions, meningitis, delirium, hyperpyrexia and death. ${ }^{1}$ Retinal haemorrhage is an important sign of prognostic significance in cerebral malaria. ${ }^{2}$ The following is the first case, to our knowledge, of cerebral malaria complicated by subarachnoid haemorrhage.

\section{Case report}

A 41 year old lorry driver was admitted with a 6-day history of fever, chills, rigors, headache and profuse sweating. On the sixth day of his illness the patient felt weak and fainted. There was no history of hypertension in the past. On admission he was febrile with a temperature of $40^{\circ} \mathrm{C}$, slightly drowsy and mildly jaundiced with hepatosplenomegaly. The central nervous system examination was normal. Examination of the optic fundi showed numerous bilateral retinal haemorrhages. While he was being taken to the X-ray department he developed cardiorespiratory arrest and was subsequently ventilated. His haemoglobin was $11.9 \mathrm{~g} / \mathrm{dl}$, white cell count $9.9 \times 10^{9} / 1$ with $86 \%$ neutrophils, platelets $80,000 \times 10^{9} / 1$, reticulocyte count $1 \%$, blood urea $10 \mathrm{mmol} / 1$, sodium $130 \mathrm{mmol} / 1$, potassium $3.1 \mathrm{mmol} / \mathrm{l}$, serum bilirubin $55 \mu \mathrm{mol} /$ litre with indirect bilirubin $45 \mu \mathrm{mol} / \mathrm{l}$, AST 15 IU (5-40 IU) and prothrombin time was 85 seconds with a control of 22 seconds. The fibrinogen titre was low. The blood film showed $50 \%$ parasitaemia with Plasmodium falciparum and lumbar puncture showed uniformly blood stained cerebrospinal fluid. His blood pressure dropped to $80 / 60 \mathrm{mmHg}$ and dopamine infusion was

Correspondence: R.F. James, M.D., M.R.C.P., West Middlesex University Hospital, Isleworth, Middlesex TW7 6AF, UK.

Accepted: 28 November 1988 started. He also had an exchange blood transfusion with 4 units of blood. Intravenous chloroquine was given as an infusion and after 24 hours the percentage of parasitaemia dropped to $1 \%$ but he remained hypotensive and died on the second day.

At autopsy, there was extensive subarachnoid haemorrhage with bleeding into the ventricles and a haematoma measuring $3 \mathrm{~cm} \times 4 \mathrm{~cm}$ on the inferior surface of the frontal lobe in the midline. The liver and spleen were enlarged. Histology of liver and spleen showed only malarial pigments with no evidence of cirrhosis of the liver. There was no adrenal haemorrhage.

\section{Discussion}

Cerebral malaria occurs when the parasitaemia is intense, ${ }^{1}$ as in this patient. The appearance of retinal haemorrhages is described as having very important prognostic significance in cerebral malaria. ${ }^{2}$ This patient had numerous bilateral retinal haemorrhages which can also be seen in Plasmodium falciparummalaria due to acute vitamin B12 or folate deficiency. ${ }^{3}$ But in this patient the levels of vitamin B12 and folate proved normal. The presence of numerous retinal haemorrhages raised the possibility of subarachnoid haemorrhage, evidence for which was provided by lumbar puncture and confirmed at autopsy. Treatment with intravenous chloroquine was instituted as primary therapy because it is superior to quinine, if chloroquine resistance is unknown in the area. ${ }^{4}$ Treatment with steroids was not considered because of its deleterious effect in cerebral malaria. ${ }^{5}$ Exchange blood transfusion is valuable when the parasitaemia is high but despite this therapy, and dopamine infusion, hypotension persisted. At autopsy there was no identifiable source of the subarachnoid bleeding or evidence of widespread bleeding diathesis. The prolonged prothombin time with normal liver histology, 
low fibrinogen titre and low platelet count confirmed the diagnosis of disseminated intravascular coagulation and we were of the opinion that the subarachnoid haemorrhage was due to disseminated intravascular coagulation. This is the first such case; to our knowledge, to be described in cerebral malaria.

\section{References}

1. Plorde, J.J. Malaria. In: Harrisons Principles of Internal Medicine, McGraw-Hill, New York, 10th edition. 1187-1191.

2. Looareesuwan, S., Warrell, D.A., White, N.J. et al. Retinal haemorrhage, a common sign of prognostic significance in cerebral malaria. Am J Trop Med Hyg 1983, 32: 911-915.

3. Fleming, A.F. Haematological manifestations of malaria and other parasitic diseases. Clin Haematol 1981, Vol. 10: 987-988.

\section{Acknowledgements}

The authors would like to thank Dr John M. Shneerson, Consultant Physician, Newmarket and Papworth Hospital for editing this article, Dr Nachinakiniyan, Director, General Hospital Jaffna and Dr Mrs Kuthathan, Pathologist, for granting permission to publish the autopsy findings and Mary Warwick and Christina James for secretarial work.

4. Cook, G.C. Prevention and treatment of Malaria. Lancet 1988, i: $32-36$.

5. Warrell, D.A., Looareesuwan, S., Warrell, M.J. et al. Dexamethasone proves deleterious in cerebral malaria - a double blind study in 100 comatosed patients. $N$ Engl J Med 1982, 306: 313-319. 